\title{
RADIATIVE ELECTROWEAK SYMMETRY BREAKING IN TeV-SCALE STRING MODELS
}

\author{
N. KITAZAWA \\ Department of Physics, Tokyo Metropolitan University, \\ Hachioji, Tokyo 192-0397, Japan \\ E-mail: kitazawa@phys.metro-u.ac.jp
}

\begin{abstract}
We examine the possibility of necessary and inevitable radiative electroweak symmetry breaking by one-loop radiative corrections in a class of string models which are the string realization of "brane world" scenario. As an example, we consider a simple quasi-realistic model based on a D3-brane at nonsupersymmetric $\mathbf{C}^{3} / \mathbf{Z}_{6}$ orbifold singularity, in which the electroweak Higgs doublet fields are identified with the massless bosonic modes of the open string on that D3-brane. We calculate the one-loop correction to the Higgs potential, and find that its vacuum expectation value can be realized in this specific model.
\end{abstract}

Keywords: String phenomenology; Electroweak symmetry breaking.

\section{Introduction and Motivations}

The electroweak symmetry breaking in the standard model is not necessary and inevitable. Though technicolor theories realize necessary and inevitable electroweak symmetry breaking, like the chiral symmetry breaking in QCD, it is not easy to construct simple viable models which is consistent with the experimental data. ${ }^{1,2}$ The radiative electroweak symmetry breaking scenario in viable models with $\mathrm{TeV}$-scale supersymmetry is also a candidate of the necessary and inevitable electroweak symmetry breaking, but it requires a certain level of fine tuning among parameters in models, ${ }^{3}$ which reduces the necessity and inevitability. There are many scenarios utilizing extra space dimensions, but it seems very difficult to construct theoretically well-defined concrete models with necessary and inevitable electroweak symmetry breaking.

Although much efforts have been made towards clarify the mechanism of the electroweak symmetry breaking in the framework of the quantum field theory, there are few efforts in the framework of the string theory, in 
spite of its potential as the unified theory including gravity. The radiative electroweak symmetry breaking in string models using D-branes is first examined by Antoniadis, Benakli and Quirós. ${ }^{4}$ They have analyzed a concrete string model with no supersymmetry, and have shown that some tree-level massless scalar fields (open string states) can follow non-trivial potential at one-loop level, and obtain non-zero vacuum expectation values. The scale of the vacuum expectation value is the radius of some compactified space, which is difficult to determine in the framework of perturbative (even nonperturbative) string theory.

The author has pointed out the possibility of the radiative electroweak symmetry breaking in which the scale of the vacuum expectation value is determined by the string scale (string tension). ${ }^{5}$ This necessary and inevitable electroweak symmetry breaking can happen in a class of nonsupersymmetric $\mathrm{TeV}$-scale string models which is constructed by "bottomup approach". ${ }^{6}$

In the following, the model of Ref.5 is very briefly introduced, and discuss possibility and problems towards constructing realistic models.

\section{A Quasi-Realistic Model}

The model is based on ten-dimensional type IIB superstring theory. The six space dimensions are considered to be compactified to some orbifold space which is the toroidal compactification with some identifications of points by some discrete transformations. Consider D3-branes whose all the three space directions are not compactified. In six-dimensional compactified space, the places of such D3-branes are specified by a point. In case that D3-branes are at a orbifold singular point (fixed point by some discrete transformation), it is shown that gauge-interacting chiral fermions can be realized in the four-dimensional world-volume of the D3-branes, and it is possible to construct models of particle physics (see Ref.6 and references therein). Depending on the nature of the singularity, the world on D3-branes becomes supersymmetric or non-supersymmetric.

Since many properties of the world on D3-brane are determined locally by the nature of the singularity, we may only specify the nature of the singularity without concretely specify the six-dimensional compactified space as the first step. Consider $\mathbf{C}^{3} / \mathbf{Z}_{6}$ orbifold singularity, where $\mathbf{C}$ denotes two dimensional space parameterized by one complex coordinate and $\mathbf{Z}_{6}$ denotes a special discrete rotational transformation which does not preserve the supersymmetry on the D3-branes. ${ }^{5}$ Only $\mathbf{Z}_{6}$ invariant states are survived on the D3-branes. The original massless open string states on a stack 
of $N$ D3-branes are described by the four-dimensional $\mathcal{N}=4$ supersymmetric $\mathrm{U}(N)$ Yang-Mills theory. The $\mathbf{Z}_{6}$ projection can break original $\mathrm{U}(N)$ gauge symmetry by setting non-trivial $\mathbf{Z}_{6}$ transformation property on the Chan-Paton factor of the open string on the D3-branes.

By considering a stack of $N=6$ D3-brane at a $\mathbf{C}^{3} / \mathbf{Z}_{6}$ singularity with some specific $\mathbf{Z}_{6}$ transformation properties of Chan-Paton indices, we have $\mathrm{U}(3) \times \mathrm{U}(2) \times \mathrm{U}(1)$ gauge symmetry with the following massless particle contents on the D3-branes (see Ref.5 for detail).

\begin{tabular}{cccc} 
field & $\mathrm{U}(3)$ & $\mathrm{U}(2)$ & $\mathrm{U}(1)$ \\
\hline$q_{L} \times 3$ & 3 & $2^{*}$ & 0 \\
$\bar{u}_{L} \times 3$ & $3^{*}$ & 1 & 1 \\
\hline$H \times 3$ & 1 & 2 & -1
\end{tabular}

Namely, we have three generations of left-handed quark doublets and righthanded up-type quarks and three Higgs doublet fields with the definition of the hypercharge

$$
Q_{Y} \equiv-\left(\frac{Q_{3}}{3}+\frac{Q_{2}}{2}+Q_{1}\right),
$$

where $Q_{n}(n=1,2,3)$ is the $\mathrm{U}(1)$ charges of $\mathrm{U}(n)$ on D3-brane. It is clear that this system has chiral anomalies and more chiral fermions should be introduced to become a consistent system. In the language of the string theory, we have to introduce some D7-branes to cancel Ramond-Ramond tadpoles. Introduction of D7-branes gives further massless particles and the chiral anomaly is cancelled out.

Three massless Higgs fields follow the potential

$$
V=\frac{g^{2}}{4} \sum_{r, s=1,2,3}\left(\left(H_{r}^{\dagger} H_{s}\right)\left(H_{s}^{\dagger} H_{r}\right)+\left(H_{r}^{\dagger} H_{r}\right)\left(H_{s}^{\dagger} H_{s}\right)\right)
$$

which has no flat directions ( $g$ denotes a gauge coupling). Therefore, if oneloop radiative correction gives negative mass squared, Higgs fields necessary have vacuum expectation values. These Higgs fields also have the following Yukawa couplings.

$$
\mathcal{L}_{Y}=-g \sum_{r, s, t=1,2,3} \epsilon_{r s t} \bar{u}_{R}^{r} q_{L}^{s} H^{t}+\text { h.c. }
$$

Though these Yukawa couplings do not have rich structure to generate hierarchical quark mass spectrum, the mixing of three Higgs doublet fields may realize hierarchy. Three Higgs field is not necessary equivalent at oneloop level depending on the way of introduction of D7-branes. 


\section{One-loop correction to the Higgs Potential}

The one-loop radiative correction to the Higgs mass can be calculated in string world-sheet theory. The two-point function of a Higgs state is divided by two parts

$$
A_{\mathrm{D} 3-\mathrm{D} 3}=A_{\mathrm{D} 3-\mathrm{D} 3}^{\mathrm{NS}}+A_{\mathrm{D} 3-\mathrm{D} 3}^{\mathrm{R}}
$$

where D3-D3 means the contribution of open string on the D3-branes, NS means Neveu-Schwarz sector describing boson loop contributions, and $\mathrm{R}$ means Ramond sector describing fermion loop contributions. There is a similar contribution by the open string whose one edge is on the D3-branes and another edge is on the D7-branes.

$$
A_{\mathrm{D} 3-\mathrm{D} 7}=A_{\mathrm{D} 3-\mathrm{D} 7}^{\mathrm{NS}}+A_{\mathrm{D} 3-\mathrm{D} 7}^{\mathrm{R}}
$$

Since boson and fermion loop give positive and negative contributions to the Higgs mass squared, respectively, if the fermionic contribution is larger than the bosonic one, the one-loop Higgs mass squared becomes negative.

The calculation is straightforward, but some comments must be given before presenting the result.

The one-loop diagram of the open string can be understood as the tree propagation of the closed string. The one-loop correction to the Higgs mass squared can be described as the summation of closed string propagation from D3-brane to D3- or D7-branes with different transverse momenta (transverse momenta, which are not conserved, mean the momenta in the direction perpendicular to the D3 or D7-branes). If there exist massless open string states which have tadpole coupling to D3-branes, they give infrared divergences in the zero transverse momentum limit. This is so called NS-NS tadpole problem. In the present model NS-NS tadpole problem exists in the contribution of untwisted closed string, and it is absent in the contribution of twisted closed string. Twisted closed staring is the string closed up to $\mathbf{Z}_{6}$ identifications, and untwisted closed string is the usual closed string. (There are two untwisted closed string sector and four twisted closed string sector in the present model.) The existence of NS-NS tadpole problem is understood as the signature that the assumed background geometry (or vacuum) is not the solution and some background redefinition is required..$^{7,8}$ It is difficult to solve this problem, since the formulation of the strings propagating non-trivial background is difficult. It may be possible that we can calculate physical quantity even in wrong ground state by the method of "tadpole resummation". ${ }^{9}$ At present, we simply neglect the contributions of untwisted closed string. 
There is a tachyon mode in the untwisted open string sector, which also means that the vacuum is unstable. It is expected that the model without tachyon mode can be constructed by considering special compactification or projection. Since the origin of the tachyon mode in the present model is related with the tachyon mode in type $0 \mathrm{~B}$ theory, ${ }^{10}$ and since the tachyon mode in type 0B theory is removed by a special projection (Sagnotti's type 0 'B model ${ }^{11}$ ), the model without tachyon mode is expected to be constructed by using type 0'B model.

The result of the calculation of one-loop Higgs mass squared is

$$
m^{2} \sim-\frac{g^{2}}{16 \pi^{2}} \frac{2}{\alpha^{\prime}} \frac{36 \sqrt{3}}{\pi} \int_{0}^{\infty} \frac{d s}{s} \frac{e^{-2 \pi^{2} / s}}{1-e^{-2 \pi^{2} / s}} e^{-s / 3},
$$

where the contribution of the untwisted closed string sector and the physical divergence due to the Yukawa coupling with massless fermions (see Eq.(3)) are neglected. This is an order estimate by neglecting the exponentially suppressed higher order contribution in the twisted open string sectors. The one-loop contribution to the mass squared is negative, because, technically, bosonic one-loop contributions of twisted sectors are cancelled out with each other due to the non-trivial $\mathbf{Z}_{6}$ transformation rule of Chan-Paton indices. The order of the "electroweak scale" is numerically obtained as

$$
v \sim \sqrt{-m^{2} / g^{2}} \simeq 10^{-2} \alpha^{\prime-1 / 2}
$$

where we used that the Higgs quartic coupling is given by the gauge coupling (see Eq.(2)). This is consistent with the standard expectation that the scale is essentially given by the string scale, $1 / \sqrt{\alpha^{\prime}}$, with one-loop suppression factor. This result suggests that necessary and inevitable radiative electroweak symmetry breaking is possible in this type of models with Dbranes at non-supersymmetric orbifold singularities.

It would be very interesting to explorer realistic models in this direction. If this scenario would be true, we would observe very rich phenomena in future high-energy colliders, LHC for example, which are very different from that would be expected in field theory models. ${ }^{12}$

\section{Acknowledgments}

The author would like to thank A.Sagnotti for fruitful discussions and the kind hospitality during the stay in The Scuola Normale Superiore in Pisa. This work has been supported in part by the Selective Research Fund of Tokyo Metropolitan University. 


\section{References}

1. M.E. Peskin and T. Takeuchi, Phys. Rev. Lett 65, 964 (1990).

2. M.E. Peskin and T. Takeuchi, Phys. Rev. D46, 381 (1992).

3. G.L. Kane et al., Phys. Lett. B551 146 (2003).

4. I. Antoniadis, K. Benakli and M. Quirós, Nucl. Phys. B583 35 (2000).

5. N. Kitazawa, Nucl. Phys. B755 254 (2006).

6. G. Aldazabal, L.E. Ibanez, F. Quevedo and A.M. Uranga, JHEP 0008002 (2000).

7. W. Fischler and L .Susskind, Phys. Lett. B171 383 (1986).

8. W. Fischler and L .Susskind, Phys. Lett. B173 262 (1986).

9. E. Dudas, G. Pradisi, M. Nicolosi and A. Sagnotti, Nucl. Phys. B708 3 (2005).

10. A. Font and A. Hernández, Nucl. Phys. B634 51 (2002).

11. A. Sagnotti, hep-th/9509080; Nucl. Phys. Proc. Suppl. 56B 332 (1997).

12. See for example, A. Cullen, M. Perelstein and M.E. Peskin, Phys. Rev. D62 055012 (2000). 